\title{
SOME REMARKS ON RATIONAL PERIODIC POINTS
}

\author{
SHu KaWAGUCHI
}

\begin{abstract}
Let $M$ be a finitely generated field over $\mathbb{Q}$ and $X$ a variety defined over $M$. We study when the set $\left\{P \in X(K) \mid f^{\circ n}(P)=P\right.$ for some $\left.n \geq 1\right\}$ is finite for any finite extension fields $K$ of $M$ and for any dominant $K$-morphisms $f: X \rightarrow X$ with $\operatorname{deg} f \geq 2$.
\end{abstract}

\section{Introduction}

To define a variety, we infer an integral separated scheme of finite type over a ground field. Let $M$ be a finitely generated field over $\mathbb{Q}$ and $X$ a variety defined over $M$. Let $K$ be a finite extension field of $M$ and $f: X \rightarrow X$ a dominant morphism defined over $K$. We say that a point $P \in X(K)$ is periodic with respect to $f$ if there is a positive integer $n$ with $f^{\circ n}(P)=P$. Let $X(K)_{p e r, f}$ be the set of periodic $K$-points with respect to $f$. We say that $X$ is periodically finite if $X(K)_{p e r, f}$ is a finite set for any finite extension fields $K$ of $M$ and any dominant $K$-morphisms $f: X \rightarrow X$ with $\operatorname{deg} f \geq 2$.

In this paper, we study when $X$ is periodically finite. In order to show the finiteness of $X(K)_{p e r, f}$, we introduce the set of backward $K$-orbits of $f$, denoted by $\lim _{f} X(K)$, which is defined by

$$
\varliminf_{f} X(K)=\left\{\left(x_{n}\right)_{n=0}^{\infty} \in \prod_{n=0}^{\infty} X(K) \mid f\left(x_{n+1}\right)=x_{n} \quad(n \geq 0)\right\} .
$$

It is easy to see that if $\lim _{f} X(K)$ is a finite set, then so is $X(K)_{p e r, f}$ and $\# \varliminf_{f} X(K)=\# X(K)_{p e r, f}$ (cf. Lemma 2.2).

We obtain the following results.

Theorem A (cf., Corollary 2.5 and $\S 6$ ). Let $X$ be a geometrically irreducible normal projective variety defined over a finitely generated field over $\mathbb{Q}$. Assume that the Picard number of $X$ is 1 (for example, $X$ is $\mathbb{P}^{n}$ or a geometrically irreducible normal projective curve). Then $X$ is periodically finite.

We prove this by using Northcott's finiteness theorem of height functions. More precisely, this result is a corollary of the fact that if there is an ample line bundle $L$ such that $f^{*}(L) \otimes L^{-1}$ is also ample, then $\lim _{f} X(K)$ is a finite set (Theorem 2.4).

Received May 26, 1999.

1991 Mathematics Subject Classification. Primary 14G05, Secondary 11G99.

Key words and phrases. periodic point, rational point. 
We also show the following theorems.

Theorem B (cf., Corollary 3.4 and $\S 6$ ). Let $C$ be a curve defined over a finitely generated field over $\mathbb{Q}$. Then $C$ is periodically finite.

Theorem C (cf., Theorem 4.4 and $\S 6$ ). Let $A$ be an abelian variety defined over a finitely generated field over $\mathbb{Q}$. Then $A$ is periodically finite if and only if $A$ is simple.

Theorem D (cf., Theorem 5.6 and $\S 6$ ). Let $X$ be a smooth projective surface with the non-negative Kodaira dimension such that $X$ is defined over a finitely generated field over $\mathbb{Q}$. Then $X$ is not periodically finite if and only if $X$ is one of the following types;

(i) $X$ is an abelian surface which is not simple, or

(ii) $X$ is a hyperelliptic surface.

In order to clarify the argument, $M$ is assumed to be a number field before $\S 6$, where in $\S 6$, we deal with a finitely generated field over $\mathbb{Q}$ in general.

The author would like to thank Prof. Yoshio Fujimoto for telling him his results $([10],[11])$. Also, the author would like to thank Prof. J.-L. ColliotThélène and Prof. Najmuddin Fakhruddin for their helpful comments on the first draft, and the referee for pointing out several inaccuracies in the paper. While the author was preparing this paper, he was partially supported by JSPS Research Fellowships for Young Scientists.

\section{Quick review of height theory}

In this section, we recall some properties of height functions. We refer to [13] for details. Let $h: \mathbb{P}^{n}(\overline{\mathbb{Q}}) \rightarrow \mathbb{R}$ be the logarithmic height function. Namely, for a point $x \in \mathbb{P}^{n}(\overline{\mathbb{Q}}), h(x)$ is defined by

$$
h(x)=\frac{1}{[K: \mathbb{Q}]} \sum_{v \in M_{K}} \log \left(\max _{1 \leq i \leq n}\left\{\left|x_{i}\right|_{v}\right\}\right),
$$

where $x=\left(x_{0}, x_{1}, \ldots, x_{n}\right) \in \mathbb{P}^{n}(K)$ is its coordinate over a sufficiently large number field $K$, and $M_{K}$ is the set of all places of $K$.

Now let $X$ be a projective variety defined over $\overline{\mathbb{Q}}, \phi: X \rightarrow \mathbb{P}^{n}$ a morphism over $\overline{\mathbb{Q}}$. For a point $x \in X(\overline{\mathbb{Q}})$, we define the height of $x$ with respect to $\phi$, denoted by $h_{\phi}(x)$, to be $h_{\phi}(x)=h(\phi(x))$.

Then the following theorem holds.

Theorem 1.1 (Height Machine). For every line bundle $L$ on a projective variety $X$ defined over $\overline{\mathbb{Q}}$, there exists a unique function $h_{L}: X(\overline{\mathbb{Q}}) \rightarrow \mathbb{R}$ modulo bounded functions with the following property;

(i) For any two line bundles $L_{1}, L_{2}, h_{L_{1} \otimes L_{2}}=h_{L_{1}}+h_{L_{2}}+O(1)$.

(ii) If $f: X \rightarrow Y$ is a morphism of projective varieties over $\overline{\mathbb{Q}}$, then $h_{f^{*}(L)}=$ $f^{*}\left(h_{L}\right)+O(1)$.

(iii) If $\phi: X \rightarrow \mathbb{P}^{n}$ is a morphism over $\overline{\mathbb{Q}}$, then $h_{\phi^{*}\left(\mathcal{O}_{\mathbb{P}} n(1)\right)}=h_{\phi}+O(1)$. 
We also recall some properties of height functions.

\section{Theorem 1.2.}

(i) (positiveness) If we denote $\operatorname{Supp}\left(\operatorname{Coker}\left(H^{0}(X, L) \otimes \mathcal{O}_{X}\right) \rightarrow L\right)$ by $\operatorname{Bs}(L)$, then $h_{L}$ is bounded below on $(X \backslash \operatorname{Bs}(L))(\overline{\mathbb{Q}})$.

(ii) (Northcott) Assume $L$ be ample. Then for any $d \geq 1$ and $M \geq 0$,

$$
\left\{x \in X(\overline{\mathbb{Q}}) \mid h_{L}(x) \leq M, \quad[\mathbb{Q}(x): \mathbb{Q}] \leq d\right\}
$$

is a finite set.

For Theorem 1.1, we refer to [13, Theorem 3.3]. For Theorem 1.2, we refer to [13, Corollary 3.4 and Proposition 3.5]. Although in [13] Theorem 1.1 (ii) is written for a morphism of smooth projective varieties, it also holds for not necessarily smooth projective varieties.

\section{Finiteness}

Let $X$ be a variety defined over a number field $M$. Let $K$ be a finite extension of $M$ and $f: X \rightarrow X$ a dominant morphism defined over $K$.

We say that a point $P \in X(K)$ is periodic with respect to $f$ if there is a positive integer $n$ with $f^{\circ n}(P)=P$. Let $X(K)_{p e r, f}$ be the set of periodic $K$ points with respect to $f$.

We also define the set of backward $K$-orbits of $f$, denoted by $\varliminf_{f} X(K)$, to be

$$
\varliminf_{f} X(K)=\left\{\left(x_{n}\right)_{n=0}^{\infty} \in \prod_{n=0}^{\infty} X(K) \mid f\left(x_{n+1}\right)=x_{n} \quad(n \geq 0)\right\} .
$$

We say that $X$ is periodically finite if for any finite extension fields $K$ of $M$ and for any dominant $K$-morphisms $f: X \rightarrow X$ with $\operatorname{deg} f \geq 2, X(K)_{\text {per, } f}$ is a finite set. Note that if there is no morphism $f: X \rightarrow X$ with $\operatorname{deg} f \geq 2$, then $X$ is periodically finite. For example, a variety of general type is periodically finite.

In this paper, we would like to study what kind of $X$ is periodically finite. We first remark elementary properties of $X(K)_{p e r, f}$ and $\varliminf_{f} X(K)$.

Lemma 2.1. Let $S \subset X(K)$ be a finite set and $\left(x_{n}\right)_{n=0}^{\infty} \in \varliminf_{f} X(K)$. Assume that there is a subsequence $\left(x_{n_{i}}\right)_{i=0}^{\infty}$ consisting of elements in $S$. Then $\left(x_{n}\right)_{n=0}^{\infty}$ is periodic, i.e., there is a positive integer $p$ with $x_{n+p}=x_{n}$ for $n \geq 0$. Moreover, $\left(x_{n}\right)_{n=0}^{\infty}$ is uniquely determined by $x_{0}$.

Proof. Since $S$ is a finite set, there is an element $s \in X(K)$ such that, for infinitely many $n, x_{n}$ equals to $s$. Let $\left(x_{n_{j}}\right)_{j=0}^{\infty}$ be the subsequence of $\left(x_{n}\right)_{n=0}^{\infty}$ with $x_{n_{j}}=s$ for $j \geq 0$. Let us set $p=n_{1}-n_{0}$. We show that $n_{2}-n_{1}=p$. Indeed, since $f^{\circ q}\left(x_{n_{2}}\right)=x_{n_{1}}$, if we set $q=n_{2}-n_{1}$, then we have $f^{\circ q}(s)=s$. If we assume $q>p$, then $n_{2}>n_{2}-p>n_{1}$ and $x_{n_{2}}=x_{n_{2}-p}=x_{n_{1}}=s$. This is a contradiction. If we assume $p>q$, then we similarly have a contradiction. Thus $n_{2}-n_{1}=n_{1}-n_{0}=p$. In the same way, $n_{j+1}-n_{j}=p$ for any $j \geq 0$. 
Now let us take any $n \geq 0$. We fix an $n_{j}$ with $n_{j}>n$ and set $r=n_{j}-n$. Then $n_{j}+p=n_{j+1}$ and $n_{j+1}-(n+p)=r$. Therefore, we get

$$
x_{n+p}=f^{\circ r}\left(x_{n_{j+1}}\right)=f^{\circ r}(s)=f^{\circ r}\left(x_{n_{j}}\right)=x_{n} .
$$

This shows that $\left(x_{n}\right)_{n=0}^{\infty}$ is periodic. Moreover if we divide $n$ by $p$ and write $n=q p+l$ with $0 \leq l \leq p-1$, then it is easy to see that $x_{n}=f^{\circ(p-l)}\left(x_{0}\right)$. This shows the latter assertion of the lemma.

The next lemma gives the relationship between $\lim _{f} X(K)$ and $X(K)_{p e r, f}$.

\section{Lemma 2.2.}

(i) If $P$ is a $K$-periodic point, then there is an element $\left(x_{n}\right)_{n=0}^{\infty} \in \varliminf_{f} X(K)$ such that $P=x_{0}$. By this correspondence, $X(K)_{\text {per, } f}$ can be seen as a subset of $\varliminf_{f} X(K)$. We say an element of $\varliminf_{f} X(K)$ which lies in the image of $X(K)_{\text {per }, f}$ is periodic.

(ii) If $X(K)_{p e r, f} \subsetneq \varliminf_{f} X(K)$ in the above correspondence, then $\varliminf_{f} X(K)$ is an infinite set.

(iii) If $\lim _{f} X(K)$ is a finite set, then $X(K)_{p e r, f}=\lim _{f} X(K)$ in the above correspondence. In particular, $X(K)_{\text {per, } f}$ is also a finite set.

Proof. (i) Let $f^{\circ p}(P)=P$. For any $n \geq 0$, we divide $n$ by $p$ and write $n=q p+l$ with $0 \leq l \leq p-1$. Then if we put $x_{n}=f^{\circ(p-l)}(P),\left(x_{n}\right)_{n=0}^{\infty}$ is an element of $\lim _{f} X(K)$.

(ii) Suppose $\left(x_{n}\right)_{n=0}^{\infty} \in \lim _{f} X(K)$ is not periodic. By lemma 2.1, for any fixed $m$, there are only finitely many $k$ with $x_{k}=x_{m}$. Then $\left\{\left(x_{n}\right)_{n=m}^{\infty} \mid m \geq\right.$ $0\} \subset \lim _{f} X(K)$ is an infinite set.

(iii) If $\varliminf_{f} X(K)$ is a finite set, then every $\left(x_{n}\right)_{n=0}^{\infty} \in \varliminf_{f} X(K)$ is periodic by (ii). In particular, $x_{0}$ is periodic. Therefore, the correspondence of (i) becomes bijective.

Next lemma shows that finiteness still holds if we change $f$ to some powers of $f$.

Lemma 2.3. Let $k$ be a positive integer.

(i) $X(K)_{p e r, f \circ k}$ is a finite set if and only if $X(K)_{p e r, f}$ is a finite set.

(ii) $\lim _{f^{\circ k}} X(K)$ is a finite set if and only if $\varliminf_{f} X(K)$ is a finite set.

Proof. (i) Suppose $P$ satisfies $f^{\circ m}(P)=P$. Then $P$ satisfies $\left(f^{\circ k}\right)^{\circ m}(P)=P$. This shows that $X(K)_{p e r, f}=X(K)_{p e r, f \circ \text {. }}$

(ii) We have only to prove the "only if" part. If $\varliminf_{f^{\circ k}} X(K)$ is a finite set, its elements are all periodic by Lemma 2.2(ii). Thus if we set

$$
S=\{x \in X(K) \mid
$$

there is an $\left(x_{n}\right)_{n=0}^{\infty} \in \varliminf_{f^{\circ k}} X(K)$ and an $m$ such that $\left.x=x_{m} \cdot\right\}$,

then $S$ is a finite set. Now the finiteness of $\lim _{f} X(K)$ follows from Lemma 2.1. 
Now we prove the following theorem.

Theorem 2.4. Let $X$ be a projective variety defined over a number field $K$ and $f: X \rightarrow X$ a surjective morphism defined over $K$. Let $d$ be a positive integer. Assume that there is an ample line bundle $L$ such that $f^{*}(L) \otimes L^{-1}$ is ample. Then $\bigcup_{\left[K^{\prime}: K\right] \leq d} \lim _{f} X\left(K^{\prime}\right)$ is a finite set. In particular, $\bigcup_{\left[K^{\prime}: K\right] \leq d} X\left(K^{\prime}\right)_{p e r, f}$ is also a finite set and \# $\bigcup_{\left[K^{\prime}: K\right] \leq d} \lim _{f} X\left(K^{\prime}\right)=\# \bigcup_{\left[K^{\prime}: K\right] \leq d} X\left(\bar{K}^{\prime}\right)_{p e r, f}$.

Proof. If we take a positive rational number $\epsilon^{\prime}$ which is sufficiently small, then $f^{*}(L) \otimes L^{-\left(1+\epsilon^{\prime}\right)}$ is still ample as a $\mathbb{Q}$-line bundle. Then by Theorem 1.2(i), and by the fact that $h_{f^{*}(L)}(P)-h_{L}(f(P))$ is a bounded function, we have a constant $C$ such that

$$
h_{L}(f(P))-\left(1+\epsilon^{\prime}\right) h_{L}(P) \geq C .
$$

for all $P \in X(\bar{K})$. Let us take an $\epsilon$ with $0<\epsilon<\epsilon^{\prime}$. Then there is a constant $M$ such that if $h_{L}(P)>M$, then

$$
h_{L}(f(P))-(1+\epsilon) h_{L}(P)>0 .
$$

Now let us define a set $S$ to be

$$
S=\left\{x \in X\left(K^{\prime}\right) \mid\left[K^{\prime}: K\right] \leq d \text { and } h_{L}(x) \leq M\right\} .
$$

Since $L$ is ample, $S$ is a finite set by Northcott.

In the following we show that, if $\left(x_{n}\right)_{n=0}^{\infty} \in \lim _{f} X\left(K^{\prime}\right)$, then there is a subsequence $\left(x_{n_{i}}\right)_{i=0}^{\infty}$ consisting of elements in $S$. In fact, suppose on the contrary that there is an $m$ such that, for any $n \geq m, x_{n}$ does not belong to $S$. Since $h_{L}\left(x_{n}\right)>M$ for $n \geq m$, we have

$$
\cdots<(1+\epsilon)^{2} h_{L}\left(x_{m+2}\right)<(1+\epsilon) h_{L}\left(x_{m+1}\right)<h_{L}\left(x_{m}\right) .
$$

This is a contradiction because

$$
h_{L}\left(x_{n}\right)<\frac{1}{(1+\epsilon)^{n-m}} h_{L}\left(x_{m}\right) \rightarrow 0 \quad(n \rightarrow \infty) .
$$

Now by applying Lemma 2.1 , we get that $\left(x_{n}\right)_{n=0}^{\infty}$ is periodic and uniquely determined by $x_{0}$. We also get that the number of $\bigcup_{\left[K^{\prime}: K\right] \leq d} \varliminf_{f} X\left(K^{\prime}\right)$ does not exceed the number of $S$. This proves the first assertion. The second assertion follows from Lemma 2.2.

As a corollary, we obtain the finiteness for a certain class of varieties.

Corollary 2.5. Let $X$ be a geometrically irreducible normal projective variety defined over a number field $M$. Assume that the Picard number of $X$ is 1 (for example, $X$ is $\mathbb{P}^{n}$ or a geometrically irreducible normal projective curve). Then $X$ is periodically finite.

Proof. Let $K$ be a finite extension field of $M$ and $f: X \rightarrow X$ be a surjective $K$-morphism of $\operatorname{deg} f \geq 2$. We take an arbitrary ample line bundle $L$ on $X$. Then by our hypothesis, there is a integer $d \geq 2$ such that $f^{*}(L)$ is numerically equivalent to $L^{\otimes d}$. In particular, $f^{*}(L) \otimes L^{-1}$ is ample. 
Let us keep the notation of Theorem 2.4. Assume here that $f^{*}(L)$ is linearly equivalent to $L^{\otimes d}$. In this case, due to Tate, there exists a unique height function $h_{L, f}$ such that $h_{L, f}=h_{L}+O(1)$ and that $h_{L, f}(f(P))=d h_{L}(P)(\mathrm{cf}$., $[6$, Chap 4. Proposotion 1.9] or [2, Corollary 1.1.1]). Then for any periodic points with respect to $f$, their height must be 0 with respect to $h_{L, f}$. An example for this is the following corollary.

Corollary 2.6. Let $K$ be a number field, $A$ an Abelian variety defined over $K$ and $[m]: A \rightarrow A$ the m-plication map with $m \geq 2$. Then $\lim _{[m]} A(K)$ is a finite set and the number of $\varliminf_{[m]} A(K)$ does not exceed the number of torsion K-points.

Proof. Extending $K$ if necessary, we may assume that there is an ample symmetric line bundle $L$ on $A$. Then $f^{*}(L) \simeq L^{\otimes m^{2}}$ and we can apply the theorem. In this case, if $x$ is a periodic point, then $x$ is a torsion point.

We finish this section by giving examples such that $X(K)_{p e r, f}$ is infinite.

Example 2.7. We give an example such that $X(K)_{p e r, f}$ (and thus $\lim _{f} X(K)$ ) is infinite. Let $E$ be an elliptic curve defined over a number field $K$ such that $E(K)$ is an infinite set. Let $X$ be $E \times E$ and $f: X \rightarrow X$ map $(P, Q)$ to $(P,[2](Q))$. Then $f$ is finite of degree 4 and the points of the form $(P, 0)$ are all periodic points.

Example 2.8. We give an example such that $X(K)_{p e r, f}$ is finite but $\varliminf_{f} X(K)$ is infinite. Let $E$ be an elliptic curve defined over a number field $K$ for which $E(K)$ contains non-torsion points. Let $P_{0} \in E(K)$ be a non-torsion point. Let $X$ be $E \times E$ and $f: X \rightarrow X \operatorname{map}(P, Q)$ to $\left(P+P_{0},[2](Q)\right)$. Then $f$ is finite of degree 4 and contains a sequence $\left(x_{n}\right)_{n=0}^{\infty} \in \lim _{f} X(K)$ with $x_{n}=\left(-[n]\left(P_{0}\right), 0\right)$. Thus by Lemma 2.2, $\varliminf_{f} X(K)$ is not finite. On the other hand, there are no periodic points.

We note that we can give examples similar to the above two examples by using $\mathbb{P}^{1}$.

\section{Curves}

By a curve, we mean an integral separated scheme of finite type over a ground field. In this section, we prove that a curve is periodically finite. Since there is no surjective morphism $f: C \rightarrow C$ with $\operatorname{deg} f \geq 2$ if $C$ is a smooth projective curve of genus $\geq 2$, we are mainly concerned with a curve $C$ such that $C \otimes \overline{\mathbb{Q}}$ is a reduced scheme consisting of rational curves and elliptic curves. First we prove two lemmas.

Lemma 3.1. Let $C$ be a curve defined over $\overline{\mathbb{Q}}$, and $f: C \rightarrow C$ a morphism over $\overline{\mathbb{Q}}$. Then there is a completion $\bar{C}$ of $C$ and a morphism $\bar{f}: \bar{C} \rightarrow \bar{C}$ which is an extension of $f$. 
Proof. Let us take an arbitrary complete curve $\bar{C}^{\prime}$ which is a completion of $C$ and set $T^{\prime}=\bar{C}^{\prime} \backslash C(\overline{\mathbb{Q}})$. If $t \in T^{\prime}$ is a singular point of $\bar{C}^{\prime}$, then we blow it up. Iterating this procedure, we get a completion $\bar{C}$ such that every point in $T=\bar{C} \backslash C(\overline{\mathbb{Q}})$ is a smooth point of $\bar{C}$. Now $f$ defines a rational map $\bar{f}: \bar{C} \rightarrow \bar{C}$. Since it is defined over $T$ and $C, \bar{f}$ is actually a morphism.

Lemma 3.2. Let $C$ be a curve defined over a number field $M$ which is geometrically irreducible. Then $C$ is periodically finite.

Proof. Let $K$ be a finite extension of $M$ and $f: X \rightarrow X$ a surjective morphism defined over $K$ with $\operatorname{deg} f \geq 2$. By taking a finite extension of $K$ if necessary, Lemma 3.1 indicates that there is a completion $\bar{C}$ of $C$ and a extension $\bar{f}$ of $f$ which are defined over $K$. Then $\lim _{f} C(K)$ can be seen as a subset of $\lim _{\bar{f}} \bar{C} \bar{C}(K)$. For a general point $P \in \bar{C}(\overline{\mathbb{Q}})$, let $L=\mathcal{O}_{\bar{C}}(P)$. Then, since $\operatorname{deg} \bar{f} \geq 2, f^{*}(L) \otimes$ $L^{-1}$ is ample. Thus, by Theorem 2.4, $\varliminf_{\bar{f}} \bar{C}(K)$ is a finite set. This proves the lemma.

Now we prove the following proposition.

Proposition 3.3. Let $C$ be a reduced scheme which is a chain of geometrically irreducible curves over $\overline{\mathbb{Q}}$. Let $f: C \rightarrow C$ be a surjective morphism such that, for every irreducible component $C_{i}$ of $C,\left.f\right|_{C_{i}}: C_{i} \rightarrow f\left(C_{i}\right)$ has degree $\geq 2$. Then for a number field $K \subset \overline{\mathbb{Q}}$ such that $C$ and $f$ are defined over $K, \varliminf_{f} C(K)$ is a finite set.

Proof. If $K^{\prime}$ is a extension field of $K$, then the finiteness of $\lim _{f} C\left(K^{\prime}\right)$ implies the finiteness of $\lim _{f} C(K)$. Thus to prove the proposition, we may take a finite extension of $K$ if necessary. Let $C_{1}, C_{2}, \ldots, C_{l}$ be the irreducible components of $C$. Since $f$ is surjective, the dimension of $f\left(C_{\alpha}\right)$ is 1 for every $\alpha$. Thus $f$ is seen to induce a transposition of the set $C_{1}, C_{2}, \ldots, C_{l}$. Then $f^{\circ l !}$ maps $C_{\alpha}$ to $C_{\alpha}$ for $1 \leq i \leq l$. Let us set $S=\left(\cup_{\alpha \neq \beta} C_{\alpha} \cap C_{\beta}\right)_{\text {red }}$. By Lemma 2.3, we have only to show that $\lim _{f^{\circ} \text { l! }} C(K)$ is a finite set. We may take a sufficiently large $K$, so that $C_{\alpha}$ 's and $S$ are all defined over $K$. Now let $\left(x_{n}\right)_{n=0}^{\infty} \in \varliminf_{\lim _{f} \text { ol! }} X(K)$.

Case 1 Suppose that there exists a subsequence $\left(x_{n_{i}}\right)_{i=0}^{\infty}$ consisting of elements in $S$. Then by Lemma 2.1, the number of $\left(x_{n}\right)_{n=0}^{\infty}$ in this case is finite.

Case 2 Suppose that there is no subsequence $\left(x_{n_{i}}\right)_{i=0}^{\infty}$ consisting of elements in $S$. Then there is an $\alpha$ such that every $x_{n}$ belongs to $C_{\alpha}$. By Lemma 3.1, $\varliminf_{f \circ \text { ol! }} C_{\alpha}(K)$ is a finite set. Thus the number of $\left(x_{n}\right)_{n=0}^{\infty}$ in this case is also finite.

As a corollary, we get

Corollary 3.4. Let $C$ be a curve defined over a number field $M$. Then $C$ is periodically finite.

Proof. Let $K$ be a finite extension of $M$ and $f: C \rightarrow C$ be a surjective $K$ morphism with $\operatorname{deg} f \geq 2$. Let us consider $C_{\overline{\mathbb{Q}}}$ and let $C_{1}, C_{2}, \ldots, C_{l}$ be its 
irreducible components. By abbreviation, $f$ also denotes the induced morphism $C_{\overline{\mathbb{Q}}} \rightarrow C_{\overline{\mathbb{Q}}}$. Since $C_{1}, C_{2}, \ldots, C_{l}$ are all conjugate to each other, the degree of $\left.f\right|_{C_{\alpha}}$ is greater or equal to 2 for each $1 \leq \alpha \leq l$. Now the assertion follows from Proposition 3.3.

\section{Abelian varieties}

Let $A$ be an abelian variety defined over a number field $M$. Recall that $A$ is said to be simple if $\operatorname{End}(A)_{\mathbb{Q}}$ is simple. In this section, we show that $A$ is periodically finite if and only if $A$ is simple. First we show that if an abelian variety is simple, then it is periodically finite.

Proposition 4.1. Let $A$ be a simple abelian variety defined over a number field $M$. Then $A$ is periodically finite.

Proof. Let $K$ be a finite extension field of $M$ and $f: X \rightarrow X$ a finite $K$ morphism with $\operatorname{deg} f \geq 2$. Let us set $B_{n}=\left\{P \in A(K) \mid f^{\circ n}(P)=P\right\}$. We prove the finiteness of $A(K)_{\text {per }, f}$ in two steps.

Step 1 We assume here that $f$ is a homomorphism. Let us denote by $A(K)_{\text {tor }}$ the set of $K$-valued torsion points on $A$. It is well known that $A(K)_{t o r}$ is a finite set (cf., Corollary 2.6). Since $A$ is simple and $f^{\circ n} \neq 1, B_{n}=\operatorname{Ker}\left(f^{\circ n}-1\right)(K)$ is a finite abelian group. In particular, $B_{n} \subset A(K)_{t o r}$. Thus $A(K)_{p e r, f}=\cup_{n=1}^{\infty} B_{n} \subset$ $A(K)_{\text {tor }}$ is a finite set.

Step 2 Here we treat a general $f$. If $B_{n}=\emptyset$ for $n \geq 1$, then we have nothing to prove. Thus we assume that there is an $k$ with $B_{k} \neq \emptyset$ and we shall prove $A(K)_{p e r, f}$ is a finite set. Since $A(K)_{p e r, f \circ k}=A(K)_{p e r, f}$ by Lemma 2.3, we may assume that $B_{1} \neq \emptyset$. We take $x_{0} \in B_{1}$, i.e., $f\left(x_{0}\right)=x_{0}$. We give $A$ another group structure such that the identity is $x_{0}$. We denote this abelian variety by $A^{\prime}$. Since $f$ maps $x_{0}$ to itself, $f$ is a homomorphism of $A^{\prime}$. Therefore, $A^{\prime}(K)_{p e r, f}$ is a finite set by Step 1 . Since $A$ and $A^{\prime}$ are identical as a set and thus $A(K)_{p e r, f}=A^{\prime}(K)_{p e r, f}$, we are done.

Next we show that if $A$ is not simple, then $A$ is not periodically finite. First we note the following lemma.

Lemma 4.2. Let $A$ be an abelian variety defined over a finitely generated field $M$ over $\mathbb{Q}$. Then there exists a finite extension field $K$ of $M$ such that $A(K)$ is an infinite set.

This is proven by many authors (cf., [12, Theorem 10.1], [8, Theorem 7.6]). We note that this is an easy corollary of Raynaud's theorem [9] (Manin-Mumford conjecture). Indeed, by Bertini's theorem, there is a curve $C$ of genus $\geq 2$ on $A_{\bar{M}}$. By Raynaud's theorem, $C(\bar{M}) \cap A(\bar{M})_{t o r}$ is a finite set. Thus if we take a sufficiently large extension field $K$ of $M$, then there exists a point $P \in C(K)$ which is not torsion. This proves the lemma.

Proposition 4.3. Let $A$ be an abelian variety defined over a number field $M$. If $A$ is not simple, then $A$ is not periodically finite. 
Proof. Since $A$ is not simple, there is an $\overline{\mathbb{Q}}$-isogeny $g: B \times C \rightarrow X$, where $B$ and $C$ are positive-dimensional abelian varieties. Let us set $D=\operatorname{Ker} g$, which is a finite group of order $d=\# D$.

We consider a morphism

$$
[d+1] \times[1]: B \times C \longrightarrow B \times C .
$$

Since, for a point $(b, c) \in D,([d] b,[d] c)=0$, we get $[d+1] \times[1](b, c)=(b, c)$ for any $(b, c) \in D$. In particular, $[d+1] \times[1]$ induces a morphism

$$
f: A \longrightarrow A \text {. }
$$

By the snake lemma, $\operatorname{Ker}([d+1] \times[1])=\operatorname{Ker} f$, thus $f$ is a surjective morphism with $\operatorname{deg} f \geq 2$. Now we take a finite extension field $K$ of $M$ such that $B$ and $C$ are defined over $K$ and that $C(K)$ is an infinite set. Then the infinite set

$$
g(\{(0, Q) \in B(K) \times C(K)\}),
$$

is contained in $A(K)_{\text {per,f }}$.

Combining Proposition 4.1 and Proposition 4.3, we obtain the following theorem.

Theorem 4.4. Let $A$ be an abelian variety defined over a number field. Then $A$ is periodically finite if and only if $A$ is simple.

\section{5. surfaces with non-negative Kodaira dimensions}

In this section we consider smooth projective surfaces with non-negative Kodaira dimensions.

E. Sato and Y. Fujimoto [10] [11] classified smooth projective varieties of $\operatorname{dim}=3$ with the non-negative Kodaira dimensions which has a non-trivial surjective endomorphism.

As a test case, they considered the surface case, which is as in the following.

Theorem 5.1 (E. Sato and Y. Fujimoto). If a smooth projective surface $X$ has a surjective endomorphism $f: X \rightarrow X$ with $\operatorname{deg} f \geq 2$, then $X$ must be minimal and is one of the following types;

(i) $X$ is an abelian surface,

(ii) $X$ is a hyperelliptic surface, or

(iii) The Kodaira dimension $\kappa(X)$ of $X$ is 1 and $X$ carries an elliptic fibration $\pi: X \rightarrow B$ whose singular fibers are at most multiple of the type ${ }_{m} I_{0}$ in the sense of Kodaira, where $B$ is a smooth projective curve.

Proof. For the reader's sake, we give a brief sketch of a proof.

Since $X$ has non-negative Kodaira dimension, $f: X \rightarrow X$ must be étale (cf., [4, Theorem 11.7]). Suppose there is an exceptional curve $C$ on $X$. Then the equality

$$
f^{*}(C) \cdot K_{X}=f^{*}(C) \cdot f^{*} K_{X}=-(\operatorname{deg} f)
$$


shows that there are at least two exceptional curves on $X$. Iterating this procedure, we get a contradiction.

We note that since $f$ is étale, $\chi_{\text {top }}(X)=(\operatorname{deg} f) \chi_{\text {top }}(X)$. Then $\operatorname{deg} f \geq 2$ implies $\chi_{\text {top }}(X)=0$. In the same way, we get $\chi\left(\mathcal{O}_{X}\right)=0$.

If $\kappa(X)=2$, then there are no surjective morphisms $f: X \rightarrow X$ with $\operatorname{deg} f \geq$ 2 (cf., [4, Proposition 10.10]); If $\kappa(X)=1$, then $\chi_{\text {top }}(X)=0$ indicates that $X$ has possibly only multiple singular fibers of type ${ }_{m} I_{0}$; If $\kappa(X)=0$, then $\chi\left(\mathcal{O}_{X}\right)=0$ indicates that $X$ cannot be a K3 surface nor an Enriques surface.

We determined in the previous section when an abelian surface is periodically finite. Now we study whether a surface of the case (ii) or (iii) is periodically finite.

Proposition 5.2. Let $X$ be a hyperelliptic surface defined over a number field $M$. Then $X$ is not periodically finite.

Proof. Let $E, F$ be arbitrary elliptic curves, $G$ a group of translations of $E$ which operates on $F$. According to the Bagnera-De Franchis list ([1, Liste VI.20]), all the hyperelliptic curves are one of the following types;

(i) $X \cong(E \times F) / G, G=\mathbb{Z} / 2$ operating on $F$ by $x \mapsto-x$,

(ii) $X \cong(E \times F) / G, G=\mathbb{Z} / 2 \times \mathbb{Z} / 2$ operating on $F$ by $x \mapsto-x, x \mapsto x+\epsilon$ $\left(\epsilon \in F_{2}\right)$,

(iii) $X \cong\left(E \times F_{i}\right) / G, G=\mathbb{Z} / 4$ operating on $F_{i}$ by $x \mapsto i x$, where $F_{i}=\mathbb{C} / \mathbb{Z}+i \mathbb{Z}$,

(iv) $X \cong\left(E \times F_{i}\right) / G, G=\mathbb{Z} / 4$ operating on $F_{i}$ by $x \mapsto i x$,

(v) $X \cong\left(E \times F_{\rho}\right) / G, G=\mathbb{Z} / 3$ operating on $F_{\rho}$ by $x \mapsto \rho x$, where $\rho=\frac{-1+\sqrt{-3}}{2}$ and $F_{\rho}=\mathbb{C} / \mathbb{Z}+\rho \mathbb{Z}$.

(vi) $X \cong\left(E \times F_{\rho}\right) / G, G=\mathbb{Z} / 3 \times \mathbb{Z} / 3$ operating on $F_{\rho}$ by $x \mapsto \rho x, x \mapsto x+\frac{1-\rho}{3}$

(vii) $X \cong\left(E \times F_{\rho}\right) / G, G=\mathbb{Z} / 6$ operating on $F_{\rho}$ by $x \mapsto-\rho x$.

Now we consider the case (i). In this case,

$$
[3] \times[1]: E \times F \longrightarrow E \times F
$$

induces a surjective morphism

$$
f: X \rightarrow X
$$

with $\operatorname{deg} f \geq 2$. If we take a sufficiently large finite extension field $K$ of $M$, Then the infinite set $\{(0, Q) \mid Q \in F(K)\}$ is contained in $(E \times F)(K)_{p e r,[3] \times[1]}$. Thus $X(K)_{p e r, f}$ is also an infinite set. The other cases can be treated in similar ways. In lieu of [3] $\times[1]$, we have only to consider $[g+1] \times[1]$ where $g=\# G$.

Next we treat a case of an elliptic surface. We prove the following lemma in advance.

Lemma 5.3. Let $\pi: X \rightarrow B$ be a flat morphism of projective varieties over with $\operatorname{dim} B=1$ such that all the fibers are (possibly non-reduced) abelian varieties. If the Kodaira dimension of $X$ is greater or equal to 1 , then the geometric genus of every horizontal curve is greater or equal to 2. 
Proof. Suppose there is a horizontal curve $C$ on $X$ such that the geometric genus of $C$ is 0 or 1 . Then there is an elliptic curve $B^{\prime}$ with a surjection $u: B^{\prime} \rightarrow C$. Let us set $v=u \circ \pi: B^{\prime} \rightarrow B$. Now we consider the following Cartesian product,

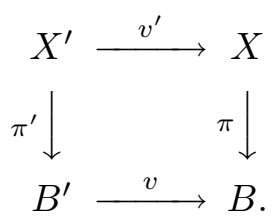

Since the singular fibers of $\pi^{\prime}$ are at most multiple fibers of an abelian variety and since $\pi^{\prime}: X^{\prime} \rightarrow B^{\prime}$ has a section, $\pi^{\prime}$ must be a smooth morphism. Then there is an elliptic curve $B^{\prime \prime}$ and an étale covering $B^{\prime \prime} \rightarrow B^{\prime}$ such that its pullback $\pi^{\prime \prime}: X^{\prime \prime}=X^{\prime} \times_{B}^{\prime} B^{\prime \prime}$ is trivial, i.e., $X^{\prime \prime}$ is a product of an abelian variety by an elliptic curve (cf. [1, Proposition VI.8]). Thus the Kodaira dimension of $X^{\prime \prime}$ is zero. On the other hand, since there is a surjective morphism $X^{\prime \prime} \rightarrow X$, the Kodaira dimension of $X^{\prime \prime}$ must be greater or equal to 1 . This is a contradiction.

Lemma 5.4. Let $K$ be a number field. Let $\pi: X \rightarrow B$ be a flat morphism of projective varieties over $K$ with $\operatorname{dim} B=1$. Let $f: X \rightarrow X$ a surjective morphism over $K$ which commutes with $\pi$, i.e., $\pi \circ f=\pi$. We make the following three assumptions.

(A) There exist a covering $u: B^{\prime} \rightarrow B$ over $K$ and an abelian variety $A$ over $K$ such that $X \times_{B} B^{\prime}$ is isomorphic to $A \times_{K} B^{\prime}$ over $B^{\prime}$ :

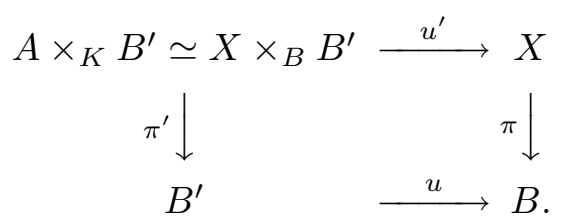

(B) If $C$ is a horizontal curve on $X$, then the geometric genus of $C$ is greater or equal to 2 .

(C) There is a line bundle $L$ on $X$ such that both $\left.L\right|_{X_{\eta}}$ and $f^{*}(L) \times\left. L^{-1}\right|_{X_{\eta}}$ are ample, where $\eta$ is a generic point of $B$.

Then $X(K)_{\text {per, } f}$ is a finite set.

Proof. We set $X^{\prime}=X \times_{B} B^{\prime}$ and $f^{\prime}=f \times_{B} i d: X^{\prime} \rightarrow X^{\prime}$. We first claim that

$$
X(K)_{p e r, f} \subset u^{\prime}\left(\bigcup_{\left[K^{\prime}: K\right] \leq d} X^{\prime}\left(K^{\prime}\right)_{p e r, f^{\prime}}\right),
$$

where $d=\operatorname{deg} u$. Indeed, for $P \in X(K)_{p e r, f}$, we set $Q=\pi(P)$ and take a point $Q^{\prime}$ on $B^{\prime}$ with $u\left(Q^{\prime}\right)=Q$. We note that $Q^{\prime}$ is defined over a number field $K^{\prime}$ with $\left[K^{\prime}: K\right] \leq d$. Then the point $P^{\prime}=\left(P, Q^{\prime}\right)$ on $X^{\prime}=X \times_{B} B^{\prime}$ satisfies $u^{\prime}\left(P^{\prime}\right)=P$ and defined over $K^{\prime}$. For $n$ with $f^{\circ n}(P)=P$, we have $f^{\prime \text { on }}\left(P^{\prime}\right)=\left(f^{\circ n}(P), Q\right)=(P, Q)=P^{\prime}$. Thus $P^{\prime} \in X^{\prime}\left(K^{\prime}\right)_{\text {per }, f^{\prime}}$. 
Let $p: X^{\prime}=A \times_{K} B^{\prime} \rightarrow A, q: X^{\prime}=A \times_{K} B^{\prime} \rightarrow B^{\prime}$ be the projections. We next claim that there are finite points $x_{1}, x_{2}, \cdots, x_{n} \in A(\bar{K})$ such that

$$
\bigcup_{\left[K^{\prime}: K\right] \leq d} X^{\prime}\left(K^{\prime}\right)_{p e r, f^{\prime}} \subset\left\{x_{1}, x_{2}, \cdots, x_{n}\right\} \times_{K} B^{\prime} .
$$

Indeed, by the rigidity of abelian varieties (cf., [5, Chap 8 Theorem 1]), there exist a finite extension field $K_{1}$ of $K$ and a morphism $g: A \rightarrow A$ defined over $K_{1}$ such that $f_{K_{1}}^{\prime}=g \times_{K_{1}} i d: X_{K_{1}}^{\prime} \rightarrow X_{K_{1}}^{\prime}$. (Here, $f_{K_{1}}^{\prime}=f \times_{K} K_{1}$ and $X_{K_{1}}^{\prime}=X^{\prime} \times_{K} K_{1}$.) Now if we set $e=\left[K_{1}: K\right]$, then we have

$$
p\left(\bigcup_{\left[K^{\prime}: K\right] \leq d} X^{\prime}\left(K^{\prime}\right)_{p e r, f^{\prime}}\right) \subset \bigcup_{\left[K^{\prime \prime}: K\right] \leq d e} A\left(K^{\prime \prime}\right)_{p e r, g} .
$$

On the other hand, if we set $L^{\prime}=\left.u^{\prime *}(L)\right|_{A}$, then, by the assumption (C), both $L^{\prime}$ and $g^{*}\left(L^{\prime}\right) \otimes L^{\prime-1}$ are ample. Thus, by Theorem $2.4, \bigcup_{\left[K^{\prime \prime}: K\right] \leq d e} A\left(K^{\prime \prime}\right)_{p e r, g}$ is a finite set. Therefore, we get the claim.

From the above two claims, we get

$$
X(K)_{p e r, f} \subset u^{\prime}\left(\left\{x_{1}, x_{2}, \cdots, x_{n}\right\} \times_{K} C^{\prime}\right) .
$$

On the other hand, $u^{\prime}\left(x_{i} \times{ }_{K} C^{\prime}\right)$ is a horizontal curve on $X$ and thus by the assumption (B), its geometric genus is greater or equal to 2 . Then $u^{\prime}\left(x_{i} \times{ }_{K} C^{\prime}\right)(K)$ is a finite set by Mordell-Faltings' theorem. Thus $X(K)_{p e r, f}$ is a finite set.

Proposition 5.5. Let $M$ be a number field. Let $X$ be a smooth projective surface defined over $M$ with the Kodaira dimension 1 . We assume that $X$ carries an elliptic fibration $\pi: X \rightarrow B$ with at most multiple singular fibers of the type ${ }_{m} I_{0}$ in the sense of Kodaira, where $B$ is a smooth projective curve of genus 0 or 1. Then $X$ is periodically finite.

Proof. Let $f: X \rightarrow X$ be a surjective morphism with $\operatorname{deg} f \geq 2$. Since $X$ has a unique structure of an elliptic fibration up to isomorphisms, there is an automorphism $g: B \rightarrow B$ with $\pi \circ f=g \circ \pi$. Let $K$ be a sufficiently large number field such that $X, B, f, \pi, g$ are all defined over $K$.

Case 1 Suppose that for any $k \geq 1, g^{\circ k}$ is not the identity morphism. In this case, the genus of $B$ is 0 or 1 . Let us set

$$
S=\left\{b \in B(\overline{\mathbb{Q}}) \mid g^{\circ k}(b)=b \text { for some } k \geq 1\right\} .
$$

We claim that $S$ consists at most two points. Indeed, suppose $S$ contains three points $b_{1}, b_{2}, b_{3} \in B(\overline{\mathbb{Q}})$ such that $g^{\circ k_{i}}\left(b_{i}\right)=b_{i}$ for $i=1,2,3$. Then for $k=k_{1} k_{2} k_{3}$ we get $g^{\circ k}\left(b_{i}\right)=b_{i}$ for $i=1,2,3$. Since $B$ is $\mathbb{P}^{1}$ or an elliptic curve, this shows that $g^{\circ k}$ is the identity morphism, which contradicts our assumption of Case 1.

We take $l$ such that $g^{\circ l}(b)=b$ for any $b \in S$. Now we prove the finiteness of $X(K)_{\text {per, } f}$ by showing the finiteness of $\varliminf_{f^{\circ l}} X(K)$ (cf., Lemma 2.2 and Lemma 2.3). Let $\left(x_{n}\right)_{n=0}^{\infty}$ be an element of $\varliminf_{f^{\circ} \mathrm{l}} X(K)$. Since $\pi\left(x_{n}\right)$ belongs to 
$S, x_{n}$ are all contained in the fiber $X_{\pi\left(x_{0}\right)}$. Since $f^{\circ l}$ is an étale morphism (cf., [4, Theorem 11.7]), $\lim _{\longleftarrow} f^{\circ l} \mid\left(X_{b}\right)_{\text {red }}\left(X_{b}\right)_{\text {red }}(K)$ is a finite set for $b \in S$ by Lemma 3.2. Using the finiteness of $S$, we obtain the finiteness of $\varliminf_{f^{\circ} \circ} X(K)$.

Case 2 Suppose that there is a $k \geq 1$ such that $g^{\circ k}$ is the identity morphism. To prove the finiteness of $X(K)_{p e r, f}$, we may (and will) assume by interchanging $f$ with $f^{\circ k}$ that $g$ is the identity morphism (cf., Lemma 2.3).

Now by re-taking sufficiently large $K$, we show that $X$ satisfies all the assumptions of Lemma 5.4. Indeed, a similar argument of the proof of Lemma 5.3 yields the assumption (A); The assumption (B) is a consequence of Lemma 5.3. Moreover, if we take an ample line bundle $L$ on $X$, then $L$ satisfies the assumption $(C)$, because the fiber is one-dimensional. Thus by Lemma $5.4, X(K)_{p e r, f}$ is a finite set.

Combining all the results of this section, we obtain the following theorem.

Theorem 5.6. Let $X$ be a smooth projective surface with the non-negative Kodaira dimension such that $X$ is defined over a number field. Then $X$ is not periodically finite if and only if $X$ is one of the following types;

(i) $X$ is an abelian surface which is not simple, or

(ii) $X$ is a hyperelliptic surface.

\section{Finitely generated fields over $\mathbb{Q}$}

In this section, we work over a finitely generated field over $\mathbb{Q}$. A. Moriwaki has recently constructed the theory of height functions over a finitely generated field over $\mathbb{Q}$. We first recall a part of his theory. We refer to [7] for details.

Let $K$ be a finitely generated field over $\mathbb{Q}$ with tr. $\operatorname{deg}_{\mathbb{Q}}(K)=d$. Let $B$ be a normal variety which is projective and flat over $\mathbb{Z}$ such that the field of rational functions of $B$ is $K$. Let $\bar{H}=\left(H, h_{H}\right)$ be a nef $C^{\infty}$-hermitian line bundle on $B$, i.e., $H$ is a line bundle on $B$ and $h_{H}$ is a $C^{\infty}$-hermitian line bundle such that for any curve on $C$ on $B, \widehat{\operatorname{deg}}\left(\widehat{c}_{1}\left(\left.\bar{H}\right|_{C}\right)\right) \geq 0$ (in the sense of the Arakelov geometry) and that the Chern form $\mathrm{c}_{1}(\bar{H})$ is semi-positive. There exist many such $\bar{B}=(B, \bar{H})$. We pick up a $\bar{B}$ and fix it in the following.

Now, for a point $x \in \mathbb{P}^{n}(\bar{K})$, let us define $h^{\bar{B}}(x)$ to be

$$
\begin{array}{r}
h^{\bar{B}}(x)=\sum_{\Gamma} \log \left(\max _{1 \leq i \leq n}\left\{-\operatorname{ord}_{\Gamma}\left(x_{i}\right)\right\} \widehat{\operatorname{deg}}\left(\widehat{\mathrm{c}}_{1}\left(\left.\bar{H}\right|_{\Gamma}\right)^{d}\right)\right)+ \\
\int_{B(\mathbb{C})} \log \left(\max _{1 \leq i \leq n}\left\{\left|x_{i}\right|\right\}\right) \mathrm{c}_{1}(\bar{H})^{d},
\end{array}
$$

where $x=\left(x_{0}, x_{1}, \ldots, x_{n}\right) \in \mathbb{P}^{n}\left(K^{\prime}\right)$ is its coordinate over a sufficiently large extension field $K^{\prime}$ of $K$, and $\Gamma$ runs through all prime divisors on $B$. This gives rise to a function $h^{\bar{B}}: \mathbb{P}^{n}(\bar{K}) \rightarrow \mathbb{R}$. 
Now let $X$ be a projective variety defined over $K, \phi: X \rightarrow \mathbb{P}^{n}$ a morphism over $K$. For a point $x \in X(\bar{K})$, we define the height of $x$ with respect to $\phi$, denoted by $h_{\phi}^{\bar{B}}(x)$, to be $h_{\phi}^{\bar{B}}(x)=h(\phi(x))$.

Then the following theorem holds as is the number field case (cf., [7, $33-\S 4]$ ).

Theorem 6.1. For every line bundle $L$ on a projective variety $X$ defined over $K$, there exists a unique function $h_{L}^{\bar{B}}: X(\bar{K}) \rightarrow \mathbb{R}$ modulo bounded functions with the following property;

(i) For any two line bundles $L_{1}, L_{2}, h_{L_{1} \otimes L_{2}}^{\bar{B}}=h_{L_{1}}^{\bar{B}}+h_{L_{2}}^{\bar{B}}+O(1)$.

(ii) If $f: X \rightarrow Y$ is a morphism of projective varieties over $K$, then $h_{f^{*}(L)}^{\bar{B}}=$ $f^{*}\left(h_{L}^{\bar{B}}\right)+O(1)$.

(iii) If $\phi: X \rightarrow \mathbb{P}^{n}$ is a morphism over $K$, then $h_{\phi^{*}\left(\mathcal{O}_{\mathbb{P}} n(1)\right)}^{\bar{B}}=h_{\phi}^{\bar{B}}+O(1)$.

Moreover the following properties hold.

(a) (positiveness) If we denote $\operatorname{Supp}\left(\operatorname{Coker}\left(H^{0}(X, L) \otimes \mathcal{O}_{X}\right) \rightarrow L\right)$ by $\operatorname{Bs}(L)$, then $h_{L}^{\bar{B}}$ is bounded below on $(X \backslash \operatorname{Bs}(L))(\bar{K})$.

(b) (Northcott) Assume $L$ is ample. Then for any $e \geq 1$ and $M \geq 0$,

$$
\left\{x \in X(\bar{K}) \mid h_{L}^{\bar{B}}(x) \leq M, \quad[K(x): K] \leq e\right\}
$$

is a finite set.

Aside from the Northcott finite theorem, we used Mordell-Faltings' theorem (cf., Lemma 5.4). It is known that this is also true for a finitely generated field over $\mathbb{Q}$ (cf., [3, Chapter VI]).

Now it is clear that all the results before this section also hold for a finitely generated field over $\mathbb{Q}$.

\section{References}

[1] A. Beauville, Surfaces algébriques complexes, Astérisque, No. 54, Société Mathématique de France, Paris, 1978.

[2] G.S. Call and J.H. Silverman, Canonical heights on varieties with morphisms, Compositio Math. 89 (1993), 163-205

[3] G. Faltings, G.Wüstholz, et al, Rational points, Third edition, Aspects of Mathematics, E6. Friedr. Vieweg \& Sohn, Braunschweig, 1992.

[4] S. Iitaka, Algebraic geometry, Graduate Texts in Mathematics 76, North-Holland Mathematical Library, 24, Springer-Verlag, New York-Berlin, 1982.

[5] S. Lang, Abelian varieties, Interscience Tracts in Pure and Applied Mathematics. No. 7, Interscience Publishers, Inc., New York, 1959.

[6] _ Fundamentals of Diophantine geometry, Springer-Verlag, New York-Berlin, 1983.

[7] A. Moriwaki, Arithmetic height functions over function fields, preprint (1998), math.NT/9809016.

[8] M.P. Murthy and R.G. Swan, Vector bundles over affine surfaces, Invent. Math. 36 (1976), 125-165.

[9] M. Raynaud, Courbes sur une variété abélienne et points de torsion, Invent. Math. 71 (1983), 207-233.

[10] E. Sato and Y. Fujimoto, On smooth projective threefolds with non-trivial surjective endomorphisms, Proc. Japan Acad. Ser. A Math. Sci. 74 (1998), 143-145. 
[11] in preparation.

[12] G. Fray and M. Jarden, Approximation theory and the rank of Abelian varieties over a large algebraic fields, Proc. London Math. Soc. Ser. III, 28 (1974), 112-128.

[13] J.-H. Silverman, The theory of height functions, Arithmetic geometry (Storrs, Conn., 1984), pp. 151-166, Springer-Verlag, New York, 1986.

Department of Mathematics, Faculty of Science, Kyoto University, Kyoto, 60601, JAPAN

E-mail address: kawaguch@kusm.kyoto-u.ac.jp 